Original article

\title{
Relationship between sleep disorders and erectile dysfunction, depression and quality of life in male patients with systolic heart failure
}

\author{
Sharareh Zeighami Mohammadi ${ }^{*}$, Mandana Shahparian², Habib Allah Esmaeili
}

(Received: 3 Jul 2015; Accept: 18 Dec 2015)

\begin{abstract}
Background and Purpose: Identification of the effects of sleep disturbances on the quality of life could result in proper treatment of patients with heart failure. This study aimed to investigate the relationship between sleep disorders and erectile dysfunction, depression and quality of life in male patients with systolic heart failure.

Methods: This correlational study was conducted on 100 male patients with systolic heart failure selected by convenience sampling. Data were collected using Epworth Sleepiness Scale (ESS), STOP-Bang questionnaire, Pittsburgh Sleep Quality Index (PSQI), Minnesota Living with Heart Failure questionnaire (MLHFQ), Center for Epidemiologic Studies Depression Scale (CES-D) and International Index of Erectile Function (IIEF-5). Data analysis was performed using descriptive statistics, Pearson's correlationcoefficient and linear regression model.

Results: In this study, sleep quality $(\mathrm{P}<0.001)$ and possibility of obstructive sleep apnea (OSA) $(\mathrm{P}=0.001)$ were significantly associated with depression and could explain $32.6 \%$ of variance in depression. In addition, possibility of OSA ( $\mathrm{P}=0.006)$ was associated with erectile dysfunction and could explain $12 \%$ of variance in erectile dysfunction. Also, possibility of OSA ( $\mathrm{P}=0.001)$ and sleep quality $(\mathrm{P}<0.001)$ had a statistically significant correlation with quality of life and could explain $36 \%$ of variance in quality of life.

Conclusion: According to the results of this study, poor sleep quality and sleep-disordered breathing were negatively correlated with mood, sexual function and overall quality of life in male patients with systolic heart failure. Therefore, proper screening and treatment of sleep disorders in patients with heart failure is of paramount importance.
\end{abstract}

Keywords: Depression, Erectile dysfunction, Heart failure, Quality of life, Sleep

\section{Introduction}

One-third of human life is allocated to sleeping. As a complex phenomenon, sleep is a basic physiological human need essential for preserving energy and physical well-being $(1,2)$. When we sleep, hormones such as epinephrine, serotonin and growth hormone are released. These hormones bring about chemical changes and increase cell nutrition in order to prepare the body for routine activities, cell repair, reorganization and nervous system functions, such as learning and memory processes (3).

Sleep disorders lead to hemodynamic, chemical, hormonal, neurological and psychological changes (4). According to a report, $74 \%$ of patients with heart failure suffer from a variety of sleep disorders (5). The most common sleep disorders among heart failure patients are insomnia, restless leg syndrome and sleep-disordered breathing (6). In this regard, results of one study indicated that $67 \%$ of heart

\footnotetext{
${ }^{1, *}$ Corresponding author: Department of Nursing, College of Nursing and Midwifery, Islamic Azad University, Karaj Branch, Karaj, Iran. Email: zeighami@kiau.ac.ir

${ }^{2}$ Alborz Hospital, Social Security Organization, Karaj, Iran

${ }^{3}$ Department of Public Health \& Epidemiology, School of Medicine, Mashhad University of Medical Sciences, Mashhad, Iran
} 
failure patients had sleep disturbance, while $44 \%$ suffered from daytime sleepiness (7).

Quality of life is largely influenced by the quality and quantity of sleep (8). Patients with heart failure normally have poor physical, mental and social quality of life (9). Sexual activity is another imperative determinant of physical and social health in the assessment of quality of life (10). In this regard, results of one study indicated that $84 \%$ of patients with heart failure suffered from erectile dysfunction (11).

Mental health is an inherent aspect of quality of life. In one study performed on 103 heart failure patients, $67 \%$ of the participants were diagnosed with depression (12). Moreover, several studies have confirmed the effects of sleep disorders on mental and emotional status, sexual function and quality of life of different patients (13-15).

In nursing practice, factors such as insomnia, sleep deprivation, and disturbed sleep pattern are the key elements of patient care plans. Therefore, nurses should recognize the multifactorial nature and consequences of sleep disorders for effective management of these health issues (1). Identification of the effects of sleep disturbances on the quality of life could result in proper treatment of patients with heart failure (2).

To date, several studies in Iran and other countries have investigated sleep quality, sleepiness and sleep disorders in heart failure patients. However, physical and psychological consequences of sleep disorders among male patients with heart failure remain unclear $(1,2,4,5,7,16)$.

Considering the physiological differences between men and women and the pivotal role of these factors in maintaining overall health (17), this study aimed to evaluate the relationship between sleep disorders and erectile dysfunction, depression and quality of life in male patients with systolic heart failure.

\section{Materials and Methods}

This correlational study was conducted on 100 male patients with systolic heart failure admitted to Alborz and Shahriar hospitals in Karaj and Shahriar, Iran in 2010. According to Brace et al. (2014), appropriate sample size for correlational studies includes 100 cases (18). Initially, sample size was calculated at 21 patients. Considering the effect of controlling variables, we increased the sample size to 100 participants for more precision. Inclusion criteria of the study were being married, history of heart failure (at least one year) and left ventricular ejection fraction (LVEF) of $<40 \%$.

Data were collected via interviews with participants using STOP-Bang questionnaire (SBQ), Pittsburgh Sleep Quality Index (PSQI), Epworth Sleepiness Scale (ESS), five-item International Index of Erectile Function (IIEF-5), Minnesota Living with Heart Failure questionnaire (MLHFQ), and Centre for Epidemiologic Studies Depression Scale (CES-D).

SBQ is a measurement tool with high sensitivity (83.6-100\%) used for screening patients with obstructive sleep apnea (OSA) $(19,20)$. This questionnaire consists of eight items, including snoring, tiredness, observed apnea, history of high blood pressure, body mass index (BMI) $(>35 \mathrm{~kg} /$ $\mathrm{m} 2)$, age ( $>50$ years), neck circumference $(>40 \mathrm{~cm})$ and male gender. Items are answered with "Yes=1" and " $\mathrm{No}=0$ " within the score range of $0-8$. Scores of $\geq 3$ and $<3$ are considered as high- and low-risk for OSA, respectively (20). Reliability coefficient of SBQ has been determined at 0.96 by Chung et al. (2008) $(19,20)$.

PSQI is a standard questionnaire consisting of 18 items to assess seven components of subjective sleep quality, sleep latency, sleep duration, habitual sleep efficiency, sleep disturbances, use of sleep medication, and daytime dysfunction during one month. Questions are scored based on a three-point Likert scale (score range: 0-21), and higher scores are indicative of poor sleep quality. In this questionnaire, total score of $>6$ represents poor sleep quality, and reliability of PSQI has been determined at 0.8 (21).

ESS consists of eight items to evaluate average daytime sleepiness. Each item is scored between 0-3 (score zero: would never doze, score three: high chance of dozing). In this questionnaire, total scores of 0-5, 6-10, 11-15 and 16-24 are defined as adequate sleep, mild sleepiness, moderate sleepiness and severe sleepiness, respectively. Reliability of ESS has been reported to be more than 0.7 (22). 
IIEF-5 questionnaire consists of five items scored based on a five-point Likert scale (score range: 5-25), and scores of $<21$ are indicative of erectile dysfunction. In this study, participants were categorized into the following groups based on IIEF-5 scores: severe erectile dysfunction (scores 5-10), moderate erectile dysfunction (scores 1115), mild erectile dysfunction (scores 16-20), and normal (scores 25-21). Reliability coefficient for IIEF-5 questionnaire has been determined at 0.96 by Bener et al. (23).

MLHFQ was developed by Rector in 1984 . This specialized questionnaire evaluates the effect of different treatments on the quality of life of patients. Moreover, MLHFQ examines the awareness of patients about the effects of heart failure on physical, socioeconomic and mental aspects of life. This questionnaire consists of 21 items, and each item is scored based on a five-point Likert scale (score range: 0-105). Compared to other questionnaires, MLHFQ is considered as a more reliable measurement tool. Higher total scores are indicative of poor quality of life. Accordingly, total scores of $<24,24-45$ and $>45$ are interpreted as good, moderate, and poor quality of life, respectively (24). Reliability coefficient of MLHFQ has been determined at 0.94 by Rector (25).

CES-D questionnaire consists of 20 items to measure the severity of depression, and each item is scored based on a four-point Likert scale (0 to 3 ). In this questionnaire, total scores of $<15,15-21$ and $>21$ are suggestive of normal, mild-to-moderate and severe depression, respectively (26). In one study by Clark et al., reliability coefficient of CES-D was determined at 0.85 (27).
In order to determine the scientific validity of the aforementioned questionnaires, content validity was confirmed by reviewing related scientific references. In addition, we used the test-retest method to verify the reliability coefficient of the questionnaires. To do so, the questionnaires were completed by 10 heart failure patients at two stages with a 10-day interval, and their correlation was measured as well. In the present study, Pearson's coefficient was determined at 0.78 for ESS, 0.90 for SBQ, 0.76 for PSQI, 0.78 for IIEF-5, 0.70 for MLHFQ and 0.84 for CES-D.

Study protocol was approved by the Ethics Committee of Islamic Azad University of Karaj, and permission was obtained from hospital officials. Moreover, objectives of the study were explained to participants, and written informed consent was obtained from all patients prior to the study. Patients were allowed to withdraw from the study at any time, and researchers were assured whether the study objectives were in line with the values and beliefs of participants. Also, researchers were obliged to inform the officials and participants about the results of the study.

Data analysis was performed in SPSS V.16 using descriptive statistics (mean, frequency and standard deviation), Pearson's correlation-coefficient and linear regression, and $\mathrm{P}$ value of 0.05 was considered significant.

\section{Results}

In this study, mean age of participants was $59.23 \pm 11.69$ years with age range of $34-88$ years, and mean duration of heart failure was $4.59 \pm 2.95$

Table 1. Mean scores of sleep disorders, erectile dysfunction, depression and aspects of quality of life (QOL) in male patients with systolic heart failure

\begin{tabular}{|c|c|c|c|}
\hline Variables & Mean \pm SD & Maximum & Minimum \\
\hline Possibility of Obstructive Sleep Apnea & $4.24 \pm 1.40$ & 7 & 1 \\
\hline Sleep Quality & $13.24 \pm 3.14$ & 21 & 7.06 \\
\hline Daytime Sleepiness & $7.11 \pm 3.76$ & 18 & 0 \\
\hline Erection Dysfunction & $14.02 \pm 6.26$ & 25 & 5 \\
\hline Depression & $20.31 \pm 12.06$ & 45 & 0 \\
\hline Emotional Aspect of QOL & $7.98 \pm 4.91$ & 20 & 0 \\
\hline Socioeconomic Aspect of QOL & $9.27 \pm 4.93$ & 20 & 0 \\
\hline Physical Aspect of QOL & $24.40 \pm 13.32$ & 52 & 0 \\
\hline Overall Score of QOL & $41.61 \pm 21.30$ & 88 & 0 \\
\hline
\end{tabular}


Table 2. Correlations between sleep disorders, erectile function, depression and quality of life (QOL)

\begin{tabular}{|c|c|c|c|}
\hline & Possibility of Obstructive Sleep Apnea & Sleep Quality & Daytime Sleepiness \\
\hline Erectile Function & $-0.322^{* *}$ & $-0.231^{*}$ & -0.146 \\
\hline Depression & $0.416^{* *}$ & $0.494^{* *}$ & 0.179 \\
\hline Emotional Aspect of QOL & $0.369^{* *}$ & $0.440^{* *}$ & $0.222^{*}$ \\
\hline Socioeconomic Aspect of QOL & $0.371^{* *}$ & $0.373^{* *}$ & $0.227^{*}$ \\
\hline Physical Aspect of QOL & $0.443^{* * *}$ & $0.463^{* *}$ & $0.233^{*}$ \\
\hline Overall Score of QOL & $0.456^{* *}$ & $0.486^{* *}$ & $0.249^{*}$ \\
\hline
\end{tabular}

*Significant correlation at 0.05 ;

$* *$ Significant correlation at 0.01

years with range of $1-15$ years. In $46 \%$ of the patients, economic status was at the medium level. In addition, $42 \%$ of the participants had primary education, and $51 \%$ were retired. Mean of BMI was calculated at $26.18 \pm 4.47 \mathrm{~kg} / \mathrm{m} 2$ (range: $18.52-48.44$ ), and mean of LVEF was $35.19 \pm 7.43$ (range: $10-40 \%$ ). Among the studied patients, $62 \%$ were non-smokers, and the most common accompanying diseases were hypertension (33\%) and diabetes (33\%).

Mean scores of different variables are shown in Table 1. Based on the determined cut-off point, $87 \%$ of the patients were high-risk for OSA, and $100 \%$ had total scores of $>6$, which was indicative of poor sleep quality. Moreover, $30 \%$ of the participants had adequate sleep, $53 \%$ had mild sleepiness, $12 \%$ had moderate sleepiness, and $2 \%$ had severe sleepiness.

According to the results of SBQ, $61 \%$ of the studied patients had snoring, $48 \%$ had tiredness, and $48 \%$ had observed apnea. In addition, $33 \%$ of the patients had history of high blood pressure, $4 \%$ had BMI of $>35 \mathrm{~kg} / \mathrm{m} 2,79 \%$ were aged over 50 years, and 51\% had neck circumference of $>40 \mathrm{~cm}$.

Among the studied participants, $80 \%$ had erectile dysfunction, $36 \%$ of which were severe, $26 \%$ were moderate, and $18 \%$ were reported as mild erectile dysfunction. On the other hand, 51\% of male patients with heart failure had poor quality of life, while $26 \%$ and $23 \%$ had moderate and good quality of life, respectively. Also, $37 \%$ of heart failure patients had normal depression, $16 \%$ had mild-to-moderate depression, and $47 \%$ suffered from severe depression.

Pearson's correlation-coefficient between sleep quality and erectile dysfunction, depression and quality of life in heart failure patients is presented in Table 2. Final results of linear regression models for erection dysfunction, depression and quality of life are shown in Table 3.

According to our findings, sleep quality and possibility of OSA were significantly associated with depression and could explain $32.6 \%$ of the variance in depression. Furthermore, possibility of OSA was associated with erectile dysfunction and could explain $12 \%$ of the variance in erectile dysfunction. Similarly, possibility of OSA and sleep quality had a statistically significant correlation with quality of life and could explain $36 \%$ of the variance in quality of life.

\section{Discussion}

According to the results of this study, poor sleep

Table 3. Linear regression models for correlations between sleep disorders, erectile dysfunction, depression and quality of life in male patients with systolic heart failure

\begin{tabular}{|c|c|c|c|c|}
\hline Dependent Variable & Independent Variable & $\boldsymbol{\beta}$ & $\mathbf{t}$ & P-value \\
\hline \multirow{2}{*}{$\begin{array}{l}\text { Depression } \\
\left(\mathrm{R}^{2}=0.326\right)\end{array}$} & Possibility of Obstructive Sleep Apnea & 0.300 & 3.448 & 0.001 \\
\hline & Sleep Quality & 0.408 & 4.698 & 0.000 \\
\hline \multirow{2}{*}{$\begin{array}{l}\text { Erectile Dysfunction } \\
\left(\mathrm{R}^{2}=0.125\right)\end{array}$} & Possibility of Obstructive Sleep Apnea & 0.279 & 2.819 & 0.006 \\
\hline & Sleep Quality & 0.151 & 1.528 & 0.130 \\
\hline \multirow{3}{*}{$\begin{array}{l}\text { Quality of Life } \\
\left(\mathrm{R}^{2}=0.360\right)\end{array}$} & Possibility of Obstructive Sleep Apnea & 0.315 & 3.594 & 0.001 \\
\hline & Sleep Quality & 0.383 & 4.488 & 0.000 \\
\hline & Daytime Sleepiness & 0.122 & 1.436 & 0.154 \\
\hline
\end{tabular}


quality in patients with heart failure was associated with increased prevalence of depression. In one study, Johnson et al. (2013) reported that in heart failure patients, difficulty with initiating and maintaining sleep and early morning wakefulness were correlated with the occurrence of depressive symptoms (13).

In a similar study conducted by Redeker et al, insomnia was reported to have a significant correlation with depression in patients with heart failure (28). In addition, insomnia was found to cause poor peripheral muscle perfusion, exacerbate fatigue and reduce the functional ability of patients. Factors such as orthopnea, cough and nocturia have also been reported to interfere with quality of sleep and negatively affect mood, cognition and quality of life (29).

Depression is considered as a significant predictor for mortality, poor prognosis and increased rate of hospital readmission in patients with heart failure $(30,31)$. Therefore, it could be concluded that improving the health and sleep quality of patients with heart failure will reduce the risk of depression.

In the present study, higher possibility of OSA in patients with heart failure was associated with increased prevalence of depression. In another study, Ong et al. (2009) also claimed that OSA had a significant correlation with depression (32). Furthermore, results obtained by McCall et al. suggested that oxygen desaturation caused by OSA was associated with higher severity of depression (33). In this regard, treatment of OSA with continuous positive airway pressure has been shown to enhance cognitive function and reduce emotional and depressive symptoms in the patients (34). However, findings of Dominici \& Gomes Mda were indicative of no significant correlation between OSA and depressive symptoms (35).

According to the literature, reduced serotonin levels lead to increased airway collapse during sleep, which increases the risk of depression (36). Considering the fact that depression and OSA often occur together and have the same risk factors, failure to identify and treat sleep breathing disorders is likely to increase fatigue, concentration problems and inability to perform daily tasks (35). On the other hand, these accompanying conditions are known to reduce psychomotor performance, which adds to the severity of depression (36). Therefore, it could be concluded that accurate screening, diagnosis and treatment of sleep breathing disorders in patients with heart failure could effectively decrease the severity of depressive symptoms.

According to the results of the current study, higher possibility of OSA in patients with heart failure was associated with increased erectile dysfunction. In this regard, findings of Teloken et al. (2006) indicated that heart failure patients with OSA had more complaints of erectile dysfunction (37). Other similar studies have also confirmed the association between sleep disorders and erectile dysfunction $(38,39)$.

Sexual dysfunction in men is influenced by several factors, such as vascular, hormonal and psychological disorders. In patients with OSA, factors such as increased levels of oxidative stress, endothelial impairment, decreased concentration of nitric oxide, increased levels of endothelin, impairment of the pituitary-gonadal axis, low androgen production, loss of libido, fatigue, decreased rapid eye movement sleep, increased sympathetic activity of the nervous system, peripheral neuropathy and impaired sacral reflexes have been reported to cause sexual dysfunction (40). Therefore, it could be concluded that proper examination and treatment of OSA in patients with heart failure could enhance their sexual function and quality of life.

In the current study, a significant correlation was observed between poor sleep quality and low quality of life in patients with heart failure. This finding is consistent with the results obtained by Liu et al. Accordingly, poor quality of sleep had a negative impact on physical and psychological aspects of quality of life in patients with heart failure (14). In another research, Brostrom et al. also stated that in heart failure patients, factors such as difficulty with initiating and maintaining sleep and early morning wakefulness were associated with poor quality of life (41). As such, treatment with continuous positive airway pressure could improve the quality of life in patients with heart failure (42). 
According to the model proposed by Riegel \& Weaver (2009), sleep disorders lead to cognitive problems, fatigue and reduced physical ability, which may have adverse effects on self-care behaviors of patients and exacerbate the symptoms and severity of their disease. Consequently, quality of life of patients is likely to decline dramatically (43).

Some of the limitations of the present study were the use of non-randomized sampling, small sample size, lack of control group and use of SBQ instead of polysomnography as a more specific test to evaluate OSA. Therefore, it is recommended that future research, especially case-control studies, be conducted on larger sample sizes using specific tests for more accurate assessment of OSA.

\section{Conclusion}

According to the results of this study, poor sleep quality and sleep breathing disorders were negatively associated with mood, sexual function and overall quality of life in male patients with systolic heart failure. Therefore, proper screening and treatment of sleep disorders in patients with heart failure is of paramount importance.

\section{Conflicts of interest}

None declared.

\section{Authors' contributions}

Zeighami contributed to statistical analysis as well as planning, conducting, and developing the article. Shahparian aided with planning and gathering data, and Dr. Esmaeili helped with calculating the sample size and giving advice for data analysis.

\section{Acknowledgments}

This study was extracted from a research project with code 1-11972. Hereby, we extend our gratitude to the Deputy of Research at Islamic Azad University of Karaj for the financial support of this study. We would also like to thank all the patients for their participation in this research project.

\section{References}

1. Moradi M, Mehrdad N, Nikpour S, Haghani H, Aalaa M, Sanjari M, et al. Sleep quality and associated factors among patients with chronic heart failure in Iran. Med J Islam Repub Iran 2014; 28:149.

2. Javadi N, Darvishpour A, Mehrdad N, Lakeh NM. Survey of sleep status and its related factors among hospitalized patients with heart failure. J Tehran Heart Cent 2015; 10(1):9-17.

3. Gaffari F, Zeighami MS. Frequency of daytime sleepiness in hypertensive women. Sci J Hamadan Nurs Midwifery Facul 2011; 19(1):5-13 (Persian).

4. Zeighami Mohammadi S, Shahparian M. Probability of obstructive sleep apnea in male patients with systolic heart failure and some related factors. J Nurs Midwifery Sci 2014; 1(3):32-40 (Persian).

5. Chen HM, Clark AP, Tsai LM, Chao YF. Self-reported sleep disturbance of patients with heart failure in Taiwan. Nurs Res 2009; 58(1):63-71.

6. Redeker NS. Sleep disturbance in people with heart failure: implications for self-care. J Cardiovasc Nurs 2008; 23(3):231-8.

7. Redeker NS, Stein S. Characteristics of sleep in patients with stable heart failure versus a comparison group. Heart Lung 2006; 35(4):252-61.

8. Farhadinasab AA, Azimi H. Study of patterns and subjective quality of sleep and their correlation with personality traits among medical students of Hamadam university of medical sciences. Sci J Hamadan Univ Med Sci Health Ser 2008; 15(1):11-5 (Persian).

9. Shojaei F. Quality of life in patients with heart failure. Hayat 2009; 14(2):5-13 (Persian).

10. Zeighami Mohamadi S, Shahparian M, Fahidi F, Fallah E. Sexual dysfunction in men with systolic heart failure and associated factors. J Critical Care Nurs 2012; 5(1):35-42 (Persian).

11. Schwarz ER, Kapur V, Bionat S, Rastogi S, Gupta R, Rosanio $S$. The prevalence and clinical relevance of sexual dysfunction in women and men with chronic heart failure. Int J Impot Res 2008; 20(1):85-91.

12. Pena FM, Modenesi Rde F, Piraciaba MC, Marins RM, Souza LB, Barcelos AF, et al. Prevalence and variables predictive of depressive symptoms in patients hospitalized for heart failure. Cardiol J 2011; 18(1):18-25. 
13. Johansson P, Brostr A. Insomnia is associated to depressive symptoms in patients with chronic heart failure. Open $\mathrm{J}$ Nurs 2013, 3(1):33-41.

14. Liu JC, Hung HL, Shyu YK, Tsai PS. The impact of sleep quality and daytime sleepiness on global quality of life in community-dwelling patients with heart failure. J Cardiovasc Nurs 2011; 26(2):99-105.

15. Go AS, Mozaffarian D, Roger VL, Benjamin EJ, Berry JD, Borden WB, et al. Heart disease and stroke statistics--2013 update: a report from the American Heart Association. Circulation 2013; 127(1):e6-245.

16. Moradi M, Mehrdad N, Nikpour S, Haghani H, Sharifi F. Heart failure is one of the most common cardiovascular disorders. In this disease heart is disable to perfusion. Iran J Nurs Res 2013; 8(2):18-25 (Persian).

17. Miller VM. Why are sex and gender important to basic physiology and translational and individualized medicine? Am J Physiol Heart Circ Physiol 2014; 306(6):H781-8.

18. Brace N, Kemp R, Snelger R, Lee J. SPSS for psychologist: a guide to data analysis using SPSS for windows. Hampshire: Macmillan Publishers Limited; 2014.

19. Chung F, Yegneswaran B, Liao P, Chung SA, Vairavanathan $\mathrm{S}$, Islam S, et al. STOP questionnaire: a tool to screen patients for obstructive sleep apnea. Anesthesiology 2008; 108(5):812-21.

20. Chung F, Subramanyam R, Liao P, Sasaki E, Shapiro C, Sun Y. High STOP-Bang score indicates a high probability of obstructive sleep apnoea. Br J Anaesth 2012; 108(5):768-75.

21. Izadi AF, Adib HM, Afazel MR. Quality of sleep and its related factors in the hospitalized elderly patients of Kashan hospital in 2007. Feyz 2009; 12(4):52-9 (Persian).

22. Masoodzadeh A, Zanganeh A, Shahbaznezhad L. Daytime sleepiness in medical students at Mazandaran university of medical sciences 2003. J Mazandaran Univ Med Sci 2006; 16(52):75-80 (Persian).

23. Bener A, Al-Ansari1 A, Al-Hamaq AO, Eldin IA, Afifi M. Prevalence of erectile dysfunction among hypertensiveand nonhypertensive Qatari men. Medicina (Kaunas) 2007; 43(11):870-8

24. Behlouli H, Feldman DE, Ducharme A, Frenette M, Giannetti N, Grondin F, et al. Identifying relative cutoff scores with neural networks for interpretation of the Minnesota Living with Heart Failure questionnaire. Conf Proc IEEE Eng Med Biol Soc 2009; 2009:6242-6.

25. Rector TS, Cohn JN. Assessment of patient outcome with the Minnesota Living with Heart Failure questionnaire: reliability and validity during a randomized, double-blind, placebocontrolled trial of pimobendan. Pimobendan Multicenter Research Group. Am Heart J 1992; 124(4):1017-25.

26. Sajadi M, Akbari A, Kianmehr M, Ataroudi AR. The relationship between self-care and depression in patients undergoing hemodialysis. Ofogh-E-Danesh 2008; 14(1):137 (Persian).

27. Clark CM, Mahoney JS, Clark DJ, Eriksen LR. Screening for depression in hepatitis $\mathrm{C}$ population: the reliability and validity of the center for epidemiologic studies depression scale (CES-D). J Adv Nurs 2002; 40(3):361-9.

28. Redeker NS, Jeon S, Muench U, Campbell D, Walsleben J, Rapoport DM. Insomnia symptoms and daytime function in stable heart failure. Sleep 2010; 33(9):1210-6.

29. Hayes D Jr, Anstead MI, Ho J, Phillips BA. Insomnia and chronic heart failure. Heart Fail Rev 2009; 14(3):171-82.

30. Testa G, Cacciatore F, Galizia G, Della-Morte D, Mazzella F, Gargiulo G, et al. Depressive symptoms predict mortality in elderly subjects with chronic heart failure. Eur J Clin Invest 2011; 41(12):1310-7.

31. Redwine LS, Mills PJ, Hong S, Rutledge T, Reis V, Maisel A, et al. Cardiac-related hospitalization and/or death associated with immune dysregulation and symptoms of depression in heart failure patients. Psychosom Med 2007; 69(1):23-9.

32. Ong JC, Gress JL, San Pedro-Salcedo MG, Manber R. Frequency and predictors of obstructive sleep apnea among individuals with major depressive disorder and insomnia. J Psychosom Res 2009; 67(2):135-41.

33. McCall WV, Harding D, O'Donovan C. Correlates of depressive symptoms in patients with obstructive sleep apnea. J Clin Sleep Med 2006; 2(4):424-6.

34. Ejaz SM, Khawaja IS, Bhatia S, Hurwitz TD. Obstructive sleep apnea and depression: a review. Innov Clin Neurosci 2011; 8(8):17-25.

35. Dominici M, Gomes Mda M. Obstructive sleep apnea (OSA) and depressive symptoms. Arq Neuropsiquiatr 2009; 67(1):35-9.

36. Schroder CM, O'Hara R. Depression and Obstructive Sleep Apnea (OSA). Ann Gen Psychiatry 2005; 4:13.

37. Teloken PE, Smith EB, Lodowsky C, Freedom T, Mulhall JP. Defining association between sleep apnea syndrome and erectile dysfunction. Urology 2006; 67(5):1033-7.

38. Goncalves MA, Guilleminault C, Ramos E, Palha A, Paiva T. Erectile dysfunction, obstructive sleep apnea syndrome and nasal CPAP treatment. Sleep Med 2005; 6(4):333-9. 
39. Margel D, Cohen M, Livne PM, Pillar G. Severe, but not mild, obstructive sleep apnea syndrome is associated with erectile dysfunction. Urology 2004; 63(3):545-9.

40. Zias N, Bezwada V, Gilman S, Chroneou A. Obstructive sleep apnea and erectile dysfunction: still a neglected risk fact or? Sleep Breath 2009; 13(1):3-10.

41. Brostrom A, Stromberg A, Dahlstrom U, Fridlund B. Sleep difficulties, daytime sleepiness, and health-related quality of life in patients with chronic heart failure. J Cardiovasc
Nurs 2004; 19(4):234-42.

42. Cormican LJ, Williams A. Sleep disordered breathing and its treatment in congestive heart failure. Heart 2005; 91(10):1265-70.

43. Riegel B, Weaver TE. Poor sleep and impaired self-care: towards a comprehensive model linking sleep, cognition, and heart failure outcomes. Eur J Cardiovasc Nurs 2009; 8(5):337-44. 